\title{
Detection of STRN-ALK fusion in thyroid nodules with indeterminate cytopathology facilitates papillary thyroid cancer diagnosis
}

\author{
Magdalena Jurkiewicz MD PhD ${ }^{1}$ ( ) | Adela Cimic MD $\quad$ | Vundavalli V. Murty PhD $^{1}$ | \\ Jennifer H. Kuo MD MS ${ }^{2}$ ～Susan Hsiao MD PhD ${ }^{1}$ | Ladan Fazlollahi MD ${ }^{1}$ | \\ Helen Fernandes $\mathrm{PhD}^{1}$
}

${ }^{1}$ Department of Pathology and Cell Biology, Columbia University Irving Medical Center, New York, New York

${ }^{2}$ Department of Surgery, Columbia University Irving Medical Center, New York, New York

\section{Correspondence}

Helen Fernandes, Department of Pathology and Cell Biology, Columbia University Irving Medical Center, 630 W 168th Street,

New York, NY 10032.

Email: hf2340@cumc.columbia.edu

\begin{abstract}
Thyroid cancer is the most common endocrine malignancy. Approximately $70 \%$ of cases of papillary thyroid carcinoma and $50 \%$ of poorly differentiated and anaplastic thyroid carcinoma harbor well-characterized driver mutations and chromosomal rearrangements that drive tumorigenesis. Molecular profiling has been helpful in identifying and informing follow-up strategies in tumors with more aggressive trajectories. Here, we report a case of papillary thyroid cancer (PTC) discovered in a patient with thyroid nodules with relatively benign ultrasound and fine needle aspiration (FNA) findings. Molecular testing in this patient identified a rare STRN-ALK fusion in two thyroid nodules with indeterminate and/or benign cytology. This led to the patient undergoing a thyroid lobectomy and a subsequent confirmation of papillary thyroid carcinoma upon resection. The report highlights the role of comprehensive molecular testing in thyroid lesions of indeterminate cytology.
\end{abstract}

\section{KEYWORDS}

fine needle aspiration cytology, molecular profiling, papillary thyroid carcinoma, STRN-ALK fusion

\section{1 | INTRODUCTION}

Most thyroid malignancies present as solitary thyroid nodules that can be clinically indistinguishable from benign nodules. Fine needle aspiration (FNA) cytology as a standard diagnostic procedure is a safe, costeffective and rapid tool for establishing a diagnosis and an excellent source of tumor tissue for ancillary studies. Molecular testing has been a valuable resource for diagnosis and management of patients with indeterminate cytology. ${ }^{1}$

Classification of thyroid cancer based on morphological evidence could be supported by molecular biomarkers, including markers that interrogate multiple genes using massively parallel sequencing or next-generation sequencing (NGS). Thyroid cancers are characterized by molecular alterations, such as activating/inactivating mutations, rearrangements, and copy number variations in genes responsible for cell proliferation, differentiation and apoptosis. Papillary thyroid cancer (PTC) develops through the activation of the RET/PTC-RASBRAF-ERK axis, with BRAF V600E being the major driver of classical PTC. $^{2}$ Other significant variants include TERT promoter, EIFAX mutations and RET rearrangements. ${ }^{2}$

Chromosomal rearrangements have been identified in PTC and include those involving RET, BRAF, NRTK1, NTRK3, and ALK. ${ }^{3}$ Herein we describe a patient with thyroid nodules where FNA revealed atypia of unknown significance (AUS) in one nodule and benign multinodular goiter in a second nodule. Molecular testing of both nodules identified an STRN-ALK fusion. This finding led to a diagnosis of 
PTC on resection of the affected lobe. This case demonstrates the utility of molecular profiling in aiding diagnosis and management of thyroid neoplasms with indeterminate histology.

\section{2 | CASE REPORT}

A 44 year old woman with medical history significant for ulcerative colitis, asthma, and ovarian cysts noted pain and difficulty with swallowing, along with pooling of saliva. Her relevant family history includes a maternal aunt with thyroid nodules. The patient had no personal history of radiation exposure. An ultrasound of the soft tissue of the neck and thyroid revealed a heterogeneous, complex, predominantly solid hypoechoic nodule containing round coarse calcifications and measuring $1.7 \times 1.4 \times 1.1 \mathrm{~cm}$ located at the lower pole of the right lobe of thyroid (Nodule 1). This nodule had low suspicion for malignancy according to American Thyroid Association (ATA) guidelines. ${ }^{4}$ Ultrasound evaluation also demonstrated an adjacent, more superiorly located solid, hypoechoic circumscribed right middle pole nodule measuring $1.0 \times 0.8 \times 1.2 \mathrm{~cm}$ (Nodule 2), with intermediate suspicion for malignancy according to ATA guidelines. ${ }^{4}$ An additional isoechoic solid nodule with a hypoechoic halo, measuring $0.7 \times 0.5$ $\times 0.7 \mathrm{~cm}$ was found in the mid to upper pole of the right lobe (Nodule 3). Taken together, ultrasound results were consistent with a multinodular thyroid gland with three right-sided thyroid nodules and did not raise strong concern for malignancy. At the time of presentation, relevant laboratory results including intact $\mathrm{PTH}, 25-\mathrm{OH}$ Vitamin D, TSH and Free T4 were within normal limits.

Subsequently, a fine needle aspiration (FNA) biopsy of the larger two nodules was performed. The right lower pole nodule (Nodule 1) demonstrated groups of follicular cells with focal cytological atypia, including mild nuclear enlargement, occasional nuclear grooves with a background of macrophages, inflammatory cells and scant colloid (Figure 1A). The findings were consistent with a follicular lesion of undetermined significance-Bethesda Category III with recommendation for follow up FNA. ${ }^{5}$ The specimen was routed for molecular testing. Biopsy results of the right middle pole nodule (Nodule 2) revealed clusters of follicular cells with Hurthle cell changes arranged in groups/sheets and singly in a background of macrophages and watery colloid. Many cyst lining cells were also noted (Figure 1B). These findings were consistent with nodular goiter with cystic degeneration classified as Bethesda Category II-Benign, with recommendation for clinical and sonographic follow-up. ${ }^{5}$

\section{3 | MOLECULAR ANALYSIS}

As per the molecular testing algorithm of thyroid FNA specimens at our institution, DNA extraction from formalin-fixed paraffin-embedded (FFPE) tissue was performed following the manufacturer's protocol using the DNeasyTissue kit on the QIAcube (QIAGEN). Extracted DNA from Nodule 1 underwent testing with a custom targeted Solid Tumor Panel based on Stem-Loop Inhibition Mediated Amplification

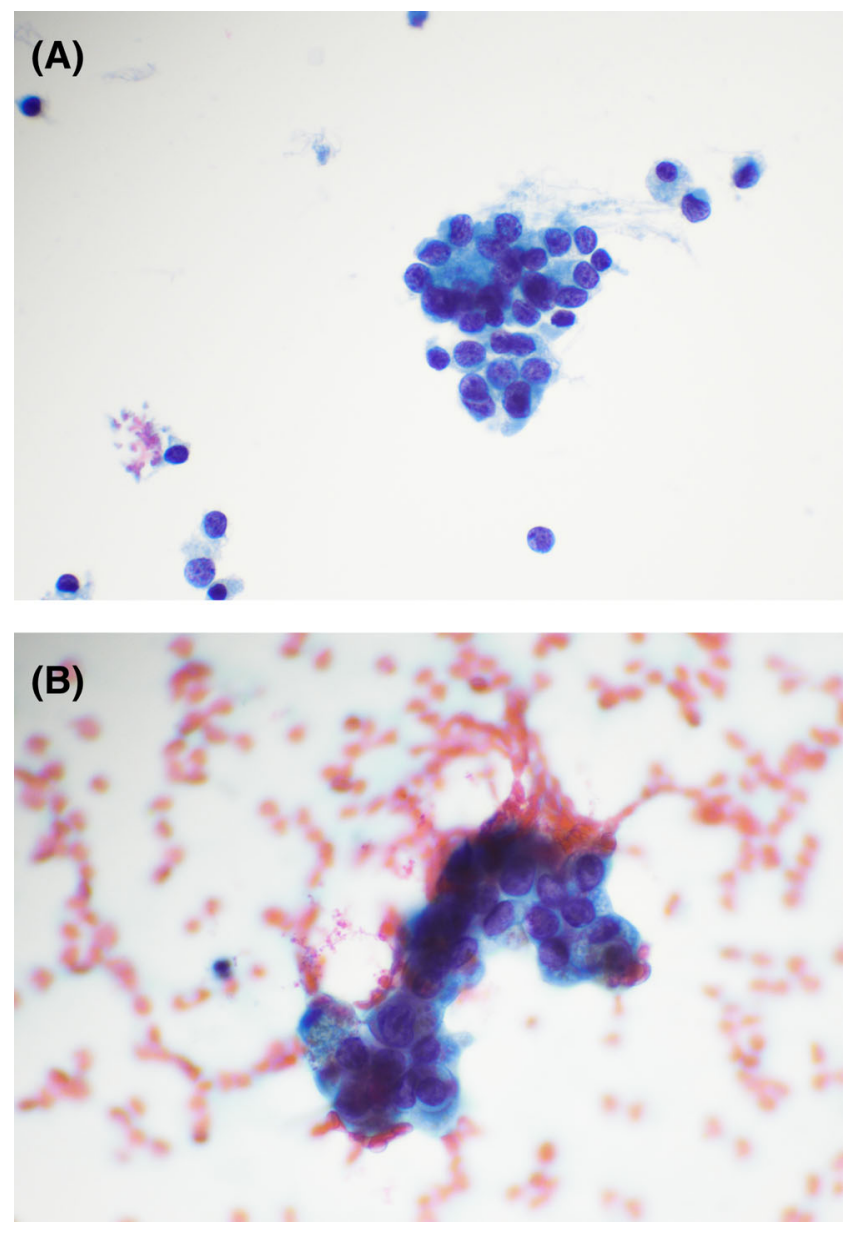

FIG URE 1 A, Nodule 1. Thyroid, right lobe, lower pole, ultrasound-guided fine needle aspiration biopsy. This specimen consists of groups of follicular cells with focal cytological atypia, including mild nuclear enlargement, occasional nuclear grooves. There is a background of macrophages, inflammatory cells, and scant colloid. These findings are consistent with a follicular lesion of undetermined significance. (H\&E, 600X). B, Nodule 2. Thyroid, right lobe, middle pole, ultrasound-guided fine needle aspiration biopsy. This specimen consists of clusters of follicular cells with Hurthle cell change arranged in groups/sheets and singly, in a background of macrophages and watery colloid. Many cyst lining cells are also noted. These findings are consistent with a nodular goiter with cystic degeneration. (H\&E, 600X)

(SLIMamp) technology (Pillar Biosciences) with multiplexed sequencing performed on the Illumina MiSeq. The following thyroid-relevant genes of the panel were interrogated for mutations: AKT1(NM_005163 e3), BRAF(NM_004333 e11,15), CTNNB1(NM_001904 e3,7,8), EIF1AX (NM_001412 e1,2,6), GNAS(NM_000516 e8,9), HRAS(NM_005343 e2-4), KRAS(NM_004985 e2-4), NRAS(NM_002524 e2-4), PIK3CA (NM_006218 e2,3,5,7,8,10,14,19,21), PTEN(NM_000314 e1-9), RET(NM_020975 e10,11,13,15,16), TERT(promoter, NM_198253 5 UTR), TP53(NM_000546 e2,4-10), TSHR(NM_000369 e9-10).

Per standard protocol at our institution, thyroid cases with no identifiable drivers in the DNA hotspot Panel are reflexed to RNA analysis from the same tissue with the Archer FusionPlex panel, which 
is a custom RNA targeted sequencing panel from Archer (Archer, Boulder, $\mathrm{CO}$ ) that aims to identify known and novel fusions in lung, thyroid, brain and gastrointestinal tumors. RNA was extracted using the ALL-Prep DNA/RNA kit (QIAGEN) followed by targeted sequencing based on Anchored Multiplex PCR (AMP) technology (Archer, Boulder, CO). cDNA libraries with molecular barcode adapters were prepared and sequencing was performed on the Illumina MiSeq platform. Identification of fusion transcripts, exons, and breakpoints was performed with the Archer Analysis software v6.0.4. Genes in the custom targeted RNA fusion panel include ALK, AXL, BRAF, EGFR, FGFR1, FGFR2, FGFR3, MET, MYB, NRG1, NTRK1, NTRK2, NTRK3, PDGRFA, PPARG, RET, ROS1, THADA. Fluorescence in situ hybridization (FISH) analysis was performed using an ALK (2p23) break-apart probe (Abbott Molecular).

\section{4 | RESULTS/FINDINGS}

The targeted DNA hotspot panel did not identify potential driver mutations in Nodule 1. As per standard protocol in our laboratory, the sample was reflexed to the RNA fusion panel. This analysis revealed a fusion between exon 3 of the striatin gene, (STRN) with the canonical anaplastic lymphoma kinase (ALK) gene breakpoint at exon 20. (Figure $2 \mathrm{~A}$ ). It was found in $42 \%$ of reads and met the quality control requirements for a confident fusion call as indicated in Table 1.

Quality control requirements for a confident fusion call were as follows: Number of supporting reads spanning the fusion junction $\geq 5$; Percentage of supporting reads spanning the fusion junction $\geq 10 \%$; Number of unique start sites (SS) for the fusion sequenced specific primer $\geq 3$. Figure $2 \mathrm{~B}$ is a representation of the STRN-ALK fusion.

(A)

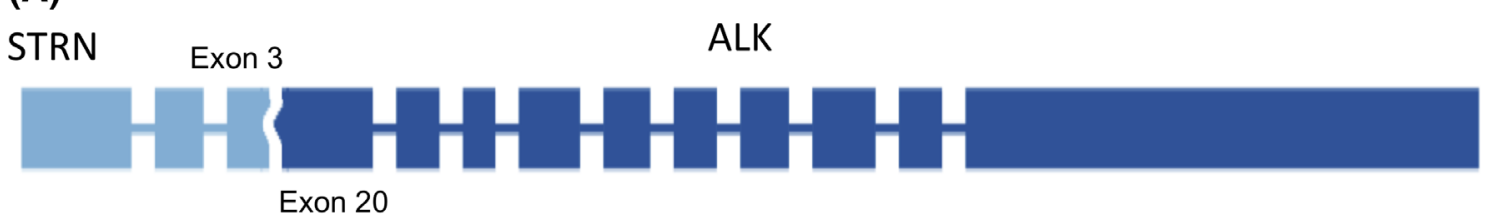

(B)

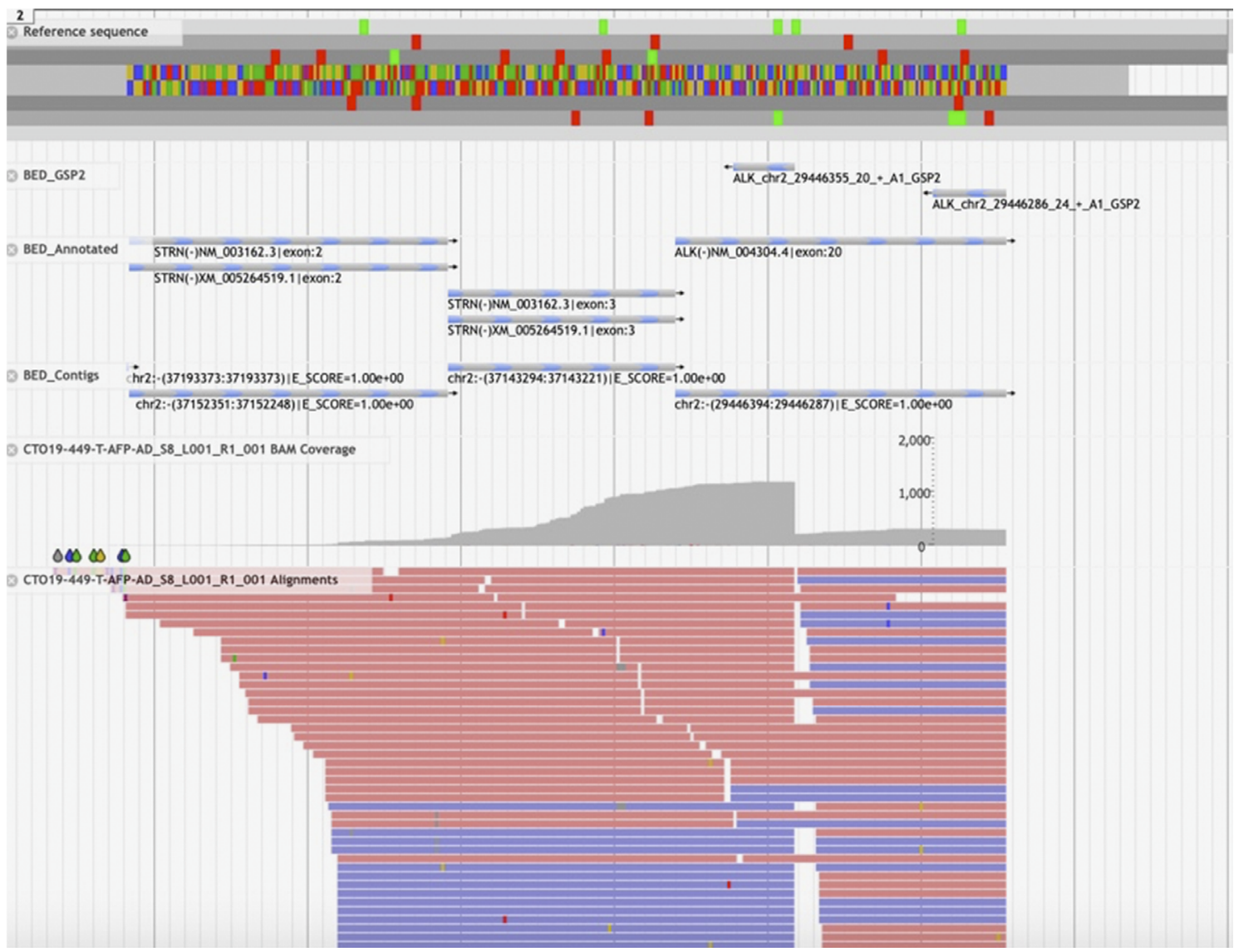

FIGURE 2 A, STRN-ALK fusion. Schematic of STRN-ALK fusion yielding E3:E20 STRN-ALK, wherein STRN is fused with the canonical breakpoint (exon 20) of the ALK gene. B, JBrowse view of the STRN-ALK fusion. The fusion between exon 3 of STRN and Exon 20 of ALK is clearly visualized in JBrowse viewer 
TABLE 1 Details for the STRN-ALK fusions found in the patient

\begin{tabular}{|c|c|c|c|c|c|c|c|}
\hline Sample & Gene 1 Accession \# & Gene 2 Accession \# & Breakpoint & Fusion sequence & $\begin{array}{l}\text { Number of } \\
\text { supporting } \\
\text { reads }\end{array}$ & $\begin{array}{l}\text { Start } \\
\text { sites }\end{array}$ & $\begin{array}{l}\% \text { of supporting } \\
\text { reads }\end{array}$ \\
\hline Nodule 1 & $\begin{array}{l}\text { STRN (exon3) } \\
\text { NM_003162.4 }\end{array}$ & $\begin{array}{l}\text { ALK (exon20) } \\
\text { NM_004304.4 }\end{array}$ & $\begin{array}{r}\text { chr2:37143221 - } \\
\text { chr2:29446394 }\end{array}$ & $\begin{array}{r}\text { GGAGATATGAAGCCTC } \\
\text { CAAGCTATGATTCTG } \\
\text { TGTACCGCCGGAAGC } \\
\text { ACCAGGAGCTGCAAG }\end{array}$ & 7 & 6 & 41.18 \\
\hline
\end{tabular}

Note: The details of the STRN-ALK fusion identified in Nodule 1 and the resection specimen are shown above, including the chromosomal breakpoint, fusion sequence, number of supporting reads and start sites. Quality control requirements for a confident fusion call are as follows: Number of supporting reads spanning the fusion junction $\geq 5$; Percentage of supporting reads spanning the fusion junction $\geq 10 \%$; Number of unique start sites (SS) for the fusion sequenced specific primer $\geq 3$.

The FNA sample from Nodule 1 was exhausted in the process of the above-mentioned testing, therefore, an FNA sample from Nodule 2 was subject to ALK rearrangement by FISH with the ALK breakapart probe. Interestingly, this nodule was positive for an ALK fusion, despite the benign histology on cytological evaluation.

Due to the rarity of the STRN-ALK fusion and previously reported strong association with malignancy, ${ }^{6}$ the patient underwent a right thyroid lobectomy. The left lobe of the thyroid showed a relatively homogenous echotexture with no nodules; therefore, a total thyroidectomy was not performed. The resection specimen revealed a histopathological diagnosis of multifocal papillary thyroid carcinoma, classical type in a background of nodular goiter (Figure 3A, B, C) with tumor, node, and metastasis (TNM) staging of T1bNO. This FFPE tissue sample showed ALK rearrangement by $\mathrm{FISH}$ in $42 \%$ of cells (Figure 4) as well as the same STRN-ALK fusion in $93 \%$ of reads. The FFPE tissue that was enriched for tumor by macrodissection had higher number of reads compared to the pauci-cellular FNA specimen in nodule 1. The patient was considered low risk for relapse and did not receive any ALK-targeted therapy. She was started on levothyroxine for thyroid hormone replacement post-operatively with a plan for regular sonographic and laboratory follow up. Six months following surgery, a hypoechoic somewhat spongiform-appearing mid to upper pole nodule measuring $0.5 \times 0.3 \times 0.5 \mathrm{~cm}$ that was not previously visualized was seen on follow-up ultrasound. Due to this nodule's small size and benign appearance, sonographic follow up was scheduled.

\section{5 | DISCUSSION}

This fusion represents an intrachromosomal rearrangement involving the short arm of chromosome 2 p22-23, resulting in a fusion of striatin (STRN), which encodes a calcium-dependent calmodulin-binding protein with the tyrosine kinase domain of anaplastic lymphoma kinase (ALK) gene. Similar to the EML4-ALK fusion, STRN-ALK fusion partners are located on the short arm of chromosome 2 (2p22.2 and
$2 \mathrm{p} 23$, separated by $\sim 7.5 \mathrm{Mb}$ ), suggesting that the fusion results from a paracentric inversion. The STRN gene includes four putative protein-protein interaction domains, which consist of a caveolinbinding domain, a coiled-coil domain, a calcium dependent calmodulin-binding domain, as well as the WD-repeat region. ${ }^{6}$ The STRN/ALK transcript is composed of the $\mathrm{N}$-terminal caveolin-binding and coiled-coil domains of STRN fused to the intracellular juxtamembrane region of ALK, which contains the tyrosine kinase domain. ${ }^{6}$ This fusion leads to constitutive activation of ALK kinase via ligandindependent dimerization mediated by the coiled-coil domain of STRN and subsequent MAPK activation. This fusion also results in a loss of the transmembrane and extracellular domains of ALK, leading to a loss of cell membrane anchoring. ${ }^{6}$ Functional studies in vitro showed that PCCL3 thyroid follicular cells transfected with STRN-ALK plasmids demonstrated increased cellular proliferation dependent on ALK kinase activity and independent of thyroid-stimulating hormone (TSH). ${ }^{6}$ In addition, transfected cells also demonstrated a spindleshaped and birefringent appearance that is suggestive of a transformed-like phenotype. in vivo experiments injecting transfected $\mathrm{NIH} 3 \mathrm{~T} 3$ cells into nude mice confirmed tumorigenicity. ${ }^{6}$

A screen of 235 cases of well-differentiated PTC revealed a $1.3 \%$ prevalence of the STRN-ALK fusion. ${ }^{6}$ The fusion occurs more often in aggressive cancers. ${ }^{6}$ None of the patients had history of radiation exposure and all lacked mutations in other known driver genes. ${ }^{6}$ This fusion has important clinical significance as the kinase activity of STRN-ALK and ALK-induced cell growth can be blocked in vitro by the ALK inhibitors crizotinib and TAE684. ${ }^{6}$ A survey of the literature shows that ALK-inhibitors are useful in treating malignancies with the STRN-ALK rearrangement. A patient with anaplastic thyroid carcinoma with infiltration beyond the thyroid capsule and four regional lymph node metastases, who was initially treated with surgery and radiochemotherapy with poor overall response was found to harbor an STRN-ALK fusion and showed an excellent clinical response to crizotinib treatment. ${ }^{7,8}$ This fusion was also noted in several patients with non-small cell lung cancer. A patient with metastatic lung adenocarcinoma was found to harbor this same STRN-ALK fusion and 

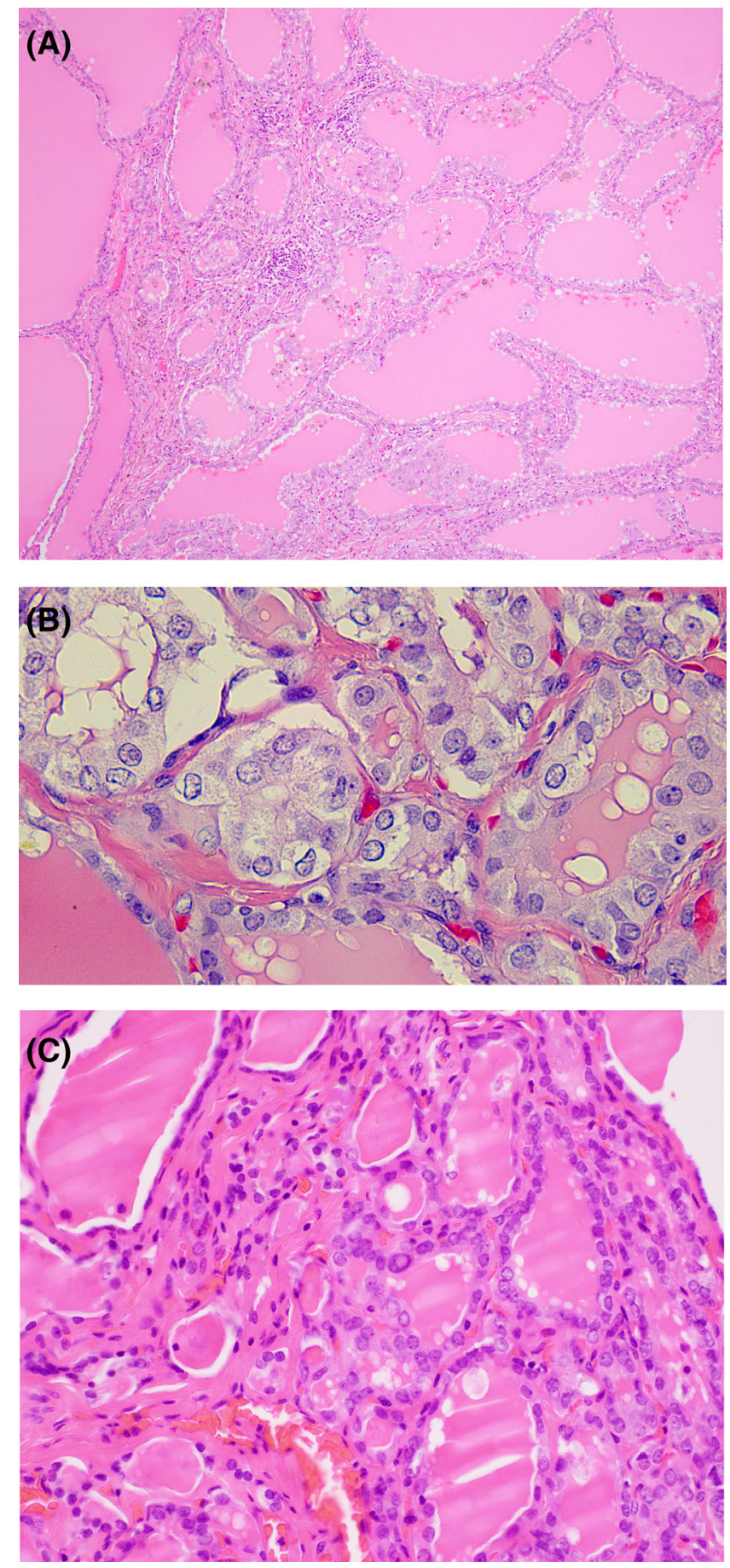

FIGURE 3 A, B, C. Thyroid gland, right lobe, lobectomy. The tumor had papillary architecture (3A) with nuclear features of papillary thyroid carcinoma such as nuclear enlargement, as well as crowding/ overlapping nuclei, nuclear grooves (3B) and nuclear pseudoinclusions (3C). Overall histologic findings are consistent with papillary thyroid carcinoma, classical type (H\&E, 2A: 100X, 2B: 400X, 2C: 600X)

showed an excellent clinical, radiographic and molecular response to treatment with crizotinib. ${ }^{10}$ In contrast, another patient with metastatic lung adenocarcinoma harboring an STRN-ALK fusion did not respond to treatment with alectinib. ${ }^{11} \mathrm{~A}$ more recent report describes

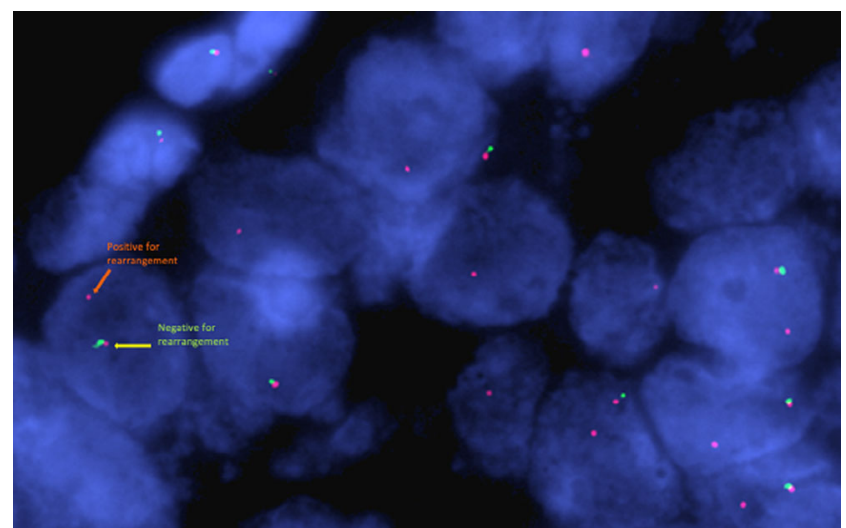

FIGURE 4 FISH analysis on FFPE tissue section using ALK breakapart probe on lobectomy sample. The fused (yellow) signal represents unrearranged normal copy of ALK (yellow arrow) and isolated reddish-orange signal is a rearranged ALK (red arrow) copy with deletion of green (5'ALK) signal

the establishment and molecular characterization of a patient-derived xenograft from a patient harboring the STRN-ALK fusion who presented with metastatic lung adenocarcinoma. ${ }^{12}$ This patient was initially treated with crizotinib with a durable response of four years until evidence of disease progression with intracerebral metastases. The patient's therapy was subsequently switched to ceritinib and whole brain radiation, which resulted in stable disease for at least 26 months at time of most recent follow up. ${ }^{12}$ Finally, a patient with malignant peritoneal mesothelioma showed dramatic response following treatment with ceritinib. ${ }^{13}$ These studies highlight the importance of identification of this particular fusion not only for diagnostic purposes, but also for therapeutic benefit. A targeted multi-gene fusion panel interrogating a list of relevant genes and enabling discovery of both known as well as novel fusions should be considered for all cases of suspected or diagnosed thyroid carcinoma due to the potential for improved diagnosis, prognosis, as well as for discovery of therapeutically targetable fusions.

\section{6 | CONCLUSIONS}

In summary, we describe a case of a patient with thyroid nodules wherein fine needle aspiration revealed atypia of unknown significance in one nodule and benign multinodular goiter in a second nodule. Subsequent molecular profiling of the FNA specimens revealed the presence of a fusion between the anaplastic lymphoma kinase (ALK) and striatin (STRN) genes. This demonstrates the utility of molecular testing as an adjunct to histopathological evaluation in diagnosis and management of thyroid malignancies, as indeterminate and even benign lesions can harbor transformative fusions with significance for patient management.

\section{CONFLICT OF INTEREST}

The authors declare no conflict of interest. 


\section{AUTHOR CONTRIBUTIONS}

$\mathrm{HF}, \mathrm{MJ}$, and $\mathrm{SH}$ analyzed and interpreted the patient's molecular findings. All authors contributed to writing and editing of the manuscript with $\mathrm{HF}$ and $\mathrm{MJ}$ as primary writers. AC provided diagnosis of and insight into the patient's cytology sample, LF provided diagnosis of and insight into the patient's thyroid lobectomy sample, VM performed cytogenetics analysis, JK treated the patient and provided clinical perspective.

\section{DATA AVAILABILITY STATEMENT}

Data sharing not applicable - no new data generated.

\section{ORCID}

Magdalena Jurkiewicz (D) https://orcid.org/0000-0002-2973-1726

\section{REFERENCES}

1. Asa SL. The current histologic classification of thyroid cancer. Endocrinol Metab Clin North Am. 2019;48(1):1-22.

2. Melillo RM, Castellone MD, Guarino V, et al. The RET/PTC-RASBRAF linear signaling cascade mediates the motile and mitogenic phenotype of thyroid cancer cells. J Clin Invest. 2005;115(4):1068-1081.

3. Bastos AU, de Jesus AC, Cerutti JM. ETV6-NTRK3 and STRN-ALK kinase fusions are recurrent events in papillary thyroid cancer of adult population. Eur J Endocrinol. 2018;178(1):83-91.

4. Floridi C, Cellina M, Buccimazza G, et al. Ultrasound imaging classifications of thyroid nodules for malignancy risk stratification and clinical management: state of the art. Gland Surg. 2019;8(Suppl 3): S233-S244.

5. Cibas ES, Ali SZ. The 2017 Bethesda system for reporting thyroid cytopathology. Thyroid. 2017;27(11):1341-1346.
6. Kelly LM, Barila G, Liu P, et al. Identification of the transforming STRN-ALK fusion as a potential therapeutic target in the aggressive forms of thyroid cancer. Proc Natl Acad Sci U S A. 2014;111(11):42334238.

7. Godbert $Y$, Henriques de Figueiredo B, Bonichon F, et al. Remarkable response to Crizotinib in woman with anaplastic lymphoma kinaserearranged anaplastic thyroid carcinoma. J Clin Oncol. 2015;33(20): e84-e87.

8. Pérot G, Soubeyran I, Ribeiro A, et al. Identification of a recurrent STRN/ALK fusion in thyroid carcinomas. PLoS One. 2014;9(1): e87170.

9. Yang Y, Qin SK, Zhu J, et al. A rare STRN-ALK fusion in lung adenocarcinoma identified using next-generation sequencing-based circulating tumor DNA profiling exhibits excellent response to Crizotinib. Mayo Clin Proc Innov Qual Outcomes. 2017;1(1):111-116.

10. Nakanishi Y, Masuda S, lida Y, Takahashi N, Hashimoto S. Case report of non-small cell lung cancer with STRN-ALK translocation: a nonresponder to alectinib. J Thorac Oncol. 2017;12(12):e202-e204.

11. Ren $\mathrm{H}$ et al. Identification and development of a lung adenocarcinoma PDX model with STRN-ALK fusion. Clinical Lung Cancer. 2019;20(2): e142-e147.

12. Rüschoff J, Gradhand E, Kahraman A, et al. STRN -ALK rearranged malignant peritoneal mesothelioma with dramatic response following Ceritinib treatment. JCO Precision Oncology. 2019;3(10):1-6.

How to cite this article: Jurkiewicz M, Cimic A, Murty VV, et al. Detection of STRN-ALK fusion in thyroid nodules with indeterminate cytopathology facilitates papillary thyroid cancer diagnosis. Diagnostic Cytopathology. 2021;49:

E146-E151. https://doi.org/10.1002/dc.24647 